\title{
Construction of 3D volumetric shape model from 2D US images
}

\author{
$\underline{\text { Mukai N. }}^{\text {a }}$, Y. Tatefuku ${ }^{\text {a }}$, M. Nakagawa ${ }^{a}$, K. Niki ${ }^{a}$ and S. Takanashi ${ }^{\text {b }}$ \\ ${ }^{a}$ Graduate School of Engineering, Tokyo City University, Japan \\ ${ }^{b}$ Cardiovascular Surgery, Sakakibara Heart Institute, Japan \\ Email:mukai@cs.tcu.ac.jp
}

\begin{abstract}
The current medical and engineering technologies help us in various fields of surgeries such as surgical simulations, preoperative plannings, intraoperative supports, and diagnostic explanations. In preoperative plannings, the simulation model of the target organ is necessary, and it is usually made from fine images that are obtained by some imaging devices such as CT (Computed Tomography) or MRI (Magnetic Resonance Imaging). However, these devices are too big to use during the surgery although there is a MRI device that can be used during the operation. On the other hand, the device of Ultrasonography (US Echo) is so handy that it can be easily used during the operation without radiation or magnetic effect; however, US images are not usually used for the construction of 3D model because they have so many noises and are not as clear as CT or MRI. Therefore, we have tried to construct 3D volumetric shape model from 2D US images by using image processing technique. Our concrete target organ and surgery are the aorta and Annuloaortic Ectasia (AAE), respectively.
\end{abstract}

In order to generate the 3D volumetric aorta model from 2D US images, two types of US images are used: the horizontal and the vertical cross section images (See Fig.1 (a) and (b)). The horizontal cross section image is obtained from the top view, which can see the circle of the aorta wall, while the vertical cross section image is obtained from the side view, which can see the aorta wall and its valve. First of all, the binary image is made from the original image by binarization process, which is one of image processing techniques. The binary image, however, has too many noises to process so that the image is thinned by contour extraction process in order to extract the aorta contour. Then, the wall and the valve of the aorta are estimated with the information of the wall, which is obtained by changing the threshold of binarization to the original images. In order to generate $2 \mathrm{D}$ clear image of the aorta, the above processes are applied to both of the horizontal and the vertical images. Finally, 3D volumetric shape model can be constructed by inserting the generated 2D horizontal image into the corresponding position of the $2 \mathrm{D}$ vertical image. In this inserting process, the horizontal image should be shrunk or expanded according to the size of the corresponding position of the vertical image.

Fig. 1 (c) and (d) show the horizontal and the vertical view of the generated 3D volumetric shape model, respectively. Although the horizontal and original 2D US image (Fig. 1 (a)) does not have the aorta valve, the generated 3D model (Fig. 1 (c)) has the valve. The generated model has $3 \mathrm{D}$ volume so that we can see the model from any point of view by rotation, and also can use it for medical applications such as surgical trainings, preoperatiave plannings, and

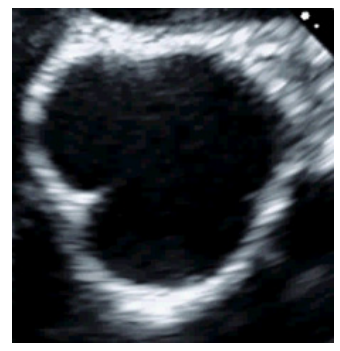

(a) horizontal US image

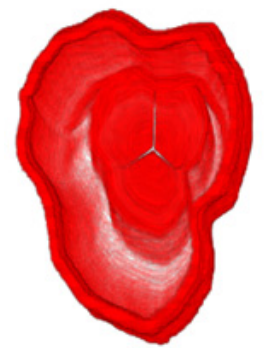

(c) horizontal view of 3D aorta model

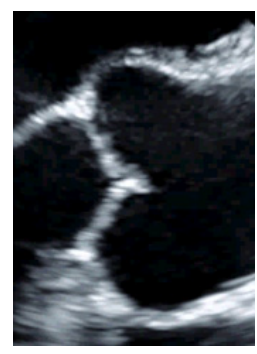

(b) vertical US image

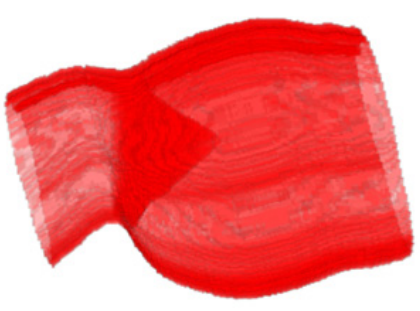

(d) vertical view of 3D aorta model

Figure 1. Original 2D US images and the generated 3D volumetric shape model.

blood flow simulations.

Keywords: Image processing, medical applications, UltraSound (US) image, aorta 


\section{INTRODUCTION}

After W. C. Roentgen invented X-ray in 1895, it has been used to generate images of human body for medical applications such as CR (Computed Radiography) and CT (Computed Tomography). X-ray is very useful to generate images of human body; however, it has a risk that the subject is radiated. Then, the new imaging technology called MRI (Magnetic Resonance Imaging) has been invented. MRI utilizes the phenomenon called NMR (Nuclear Magnetic Resonance) and does not use X-ray. CT devices can visualizes some sort of hard elements such as bones, while MRI devices can image some soft elements that include Hydrogen, such as organs and blood vessels. Especially, MRA (Magnetic Resonance Angiography) is used to generate clear images of blood vessels. In addition, PET (Positron Emission Tomography) and SPECT (Single Photon Emission Computed Tomography) are used to analyze the function of human body, while CT and MRI are used to image the human body itself. By using these images, many CAD (Computer Aided Diagnosis) systems have been developed and used for clinical applications. In addition, many image processing methods have being researched such as an automatic detection of pulmonary nodule on X-ray photos (e.g. Oda et al., 2004), an automatic detection of aneurysm on MRA image (e.g. Aoyama et al., 2010). There are also many related works such as indexing and querying techniques of medical images (Glatard et al., 2004), fast processing on medical data by using distributed and grid computing (Chrisochoides et al., 2006), and visualization of medical data with holography (Plesniak et al., 2003).

On the other hand, there is another method to check human body: ultrasonography (US Echo) that is a method to image human body by using ultrasound. Ultrasound has a long wavelength and small amount of energy so that it is not so dangerous to human body, and US Echo is a very safe and useful method to check human body, especially for pregnant women. The image, however, is not as clear as CT or MRI, and it is difficult to obtain detail medical data based on US image. There are some researches on US image such as enhancement of the resolution by adaptive beamforming (Toosi and Behnam, 2009) and interpolation of US images by using coherent signals (Trucco et al., 2009). These researches are related to improve the quality of US image and there are not methods to construct 3D medical data from US images. Therefore, we have been trying to establish a method of constructing 3D shape model for medical application, especially for the aorta surgery (Tatefuku et al., 2010). In this paper, we describe how to construct 3D volumetric shape model from 2D US images.

\section{METHOD}

Fig. 2 shows the aorta model that expands the valsalva part. In the proposed method, two types of US images are necessary: the images of the horizontal and the vertical cross sections shown in Fig. 2. By combining these two types of images, 3D volumetric shape model can be constructed. The original US images are shown in Fig. 1 (a) and (b). The flow chart of the method is shown in Fig. 3. The original images (Fig. 1 (a) and (b)) are input into the system. Then, the images are transformed into binary images by binarization process and the contours of the aorta are extracted from the binary images. These processes are applied to both of the horizontal and the vertical cross section images. Next, the aorta wall and the valve are estimated based on the contour images of the aorta. Finally, 3D aorta shape model is constructed by inserting the generated 2D horizontal image into the generated 2D vertical image. In this section, two processes of binarization and contour extraction are explained, and the estimations of the aorta wall and the valve are described in the following sections.

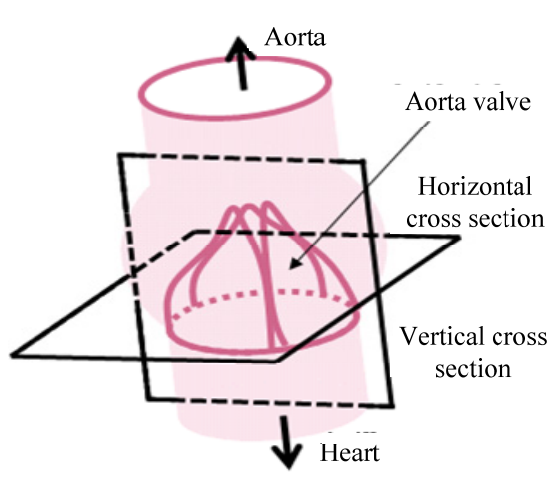

Figure 2. Aorta model.

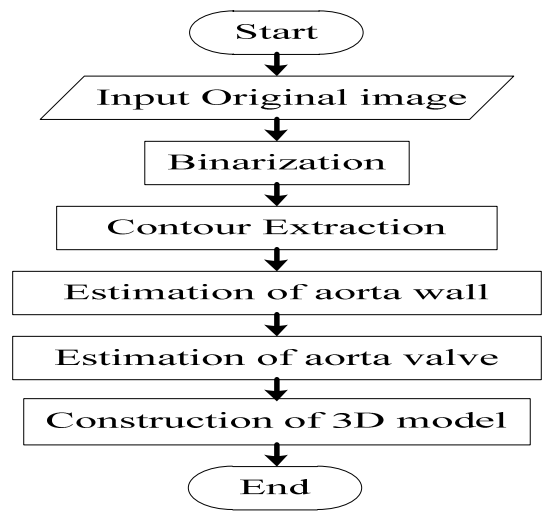

Figure 3. Flow chart of the proposed method. 
Fig. 4 shows the binary images obtained from the original images (Fig. 4 (a) and (b)) after noise reduction. The binarization thresholds for the horizontal and the vertical images were 25 and 40 respectively, where the intensity level is $[0,255]$. In this stage, our purpose is to obtain the boundary so that the threshold should be lower because the wall elements must be connected. Therefore, the walls of the mages shown in Fig. 4 are too thick because US image reflects some parts around the wall. Then, the wall thickness is estimated from the original images in the next section. Before the estimation, it is necessary to extract the boundary of the wall. Fig. 5 shows the boundary images of the aorta wall. The boundaries can be extracted by contour extraction process, where some boundaries that have short length are removed.

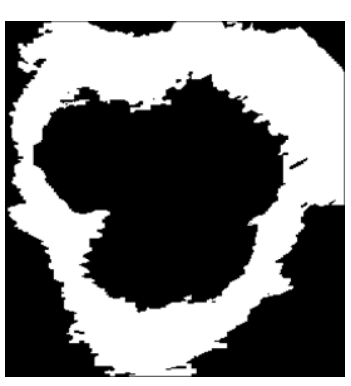

(a) horizontal image

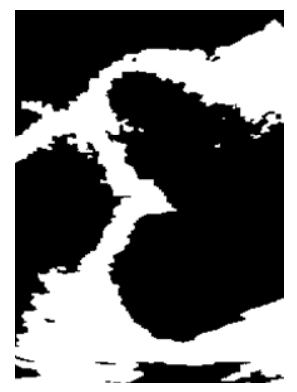

(b) vertical image

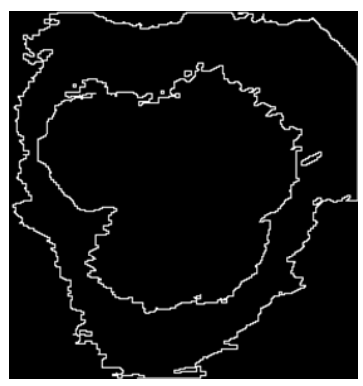

(a) horizontal image

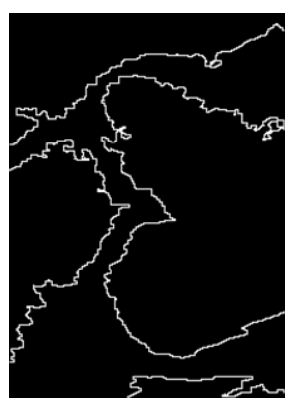

(b) vertical image

Figure 4. Binary images.

Figure 5. Boundary images.

\section{ESTIMATION OF AORTA WALL}

\subsection{Horizontal cross section image}

The aorta valve is constructed with three elements so that it is important to estimate the center of the valve. It is the best way to define the center by approximating the inner boundary of the aorta with three ellipses; however, it is difficult to divide the inner boundary into three parts. Then, a temporary center is defined as the center of gravity of an inscribed maximum triangle, as shown in Fig. 6. As mentioned before, although the aorta wall of the binary image (Fig. 4 (a)) is too thick, the actual wall is the bright area of the original image (Fig. 1 (a)). Then, we can obtain some parts of the wall by intersecting the binary image obtained by binarization with the threshold 200, and the previous binary image (Fig. 4 (a)). The obtained some parts of the aorta wall are shown in Fig. 7 (a).

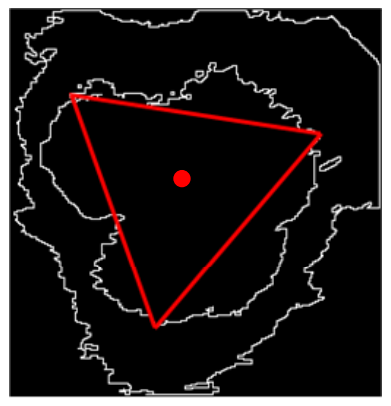

Figure 6. Inscribed maximum triangle.

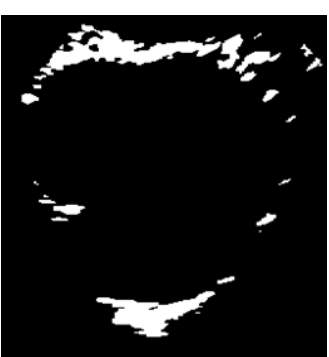

(a) binary image

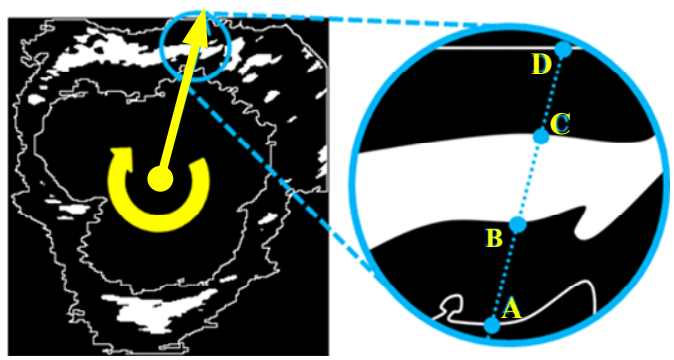

(b) thickness measurement

Figure 7. Some parts of the wall and the measurement (horizontal).

In order to estimate the thickness of the aorta wall, it is necessary to measure some parts of the wall in Fig. 7 (a). A half line extended from the temporary center is shown in Fig. 7 (b). The line is defined by connecting the center and any point of the inner boundary. Then, four points are obtained by intersecting the half line and some parts of the aorta wall (Fig. 7 (b)): A, B, C and D. Here, the wall thickness is BC and the length between the inner and the outer boundaries is $\mathrm{AD}$. Then, we can estimate the wall thickness by applying the average value of $\mathrm{BC} / \mathrm{AD}$ to each part of the wall. Here, the half line is defined clockwise around the temporary center. By smoothing the estimated wall points with Bezier curve, the aorta wall is generated. The generated wall image is shown in Fig. 8. 


\subsection{Vertical cross section image}

In the case of the vertical cross section of the aorta, the actual wall is not as clear as the horizontal cross section, and the wall is broken at some parts. Then, firstly the abstract thickness of the wall is needed to estimate the detail one. In addition, the aorta valve should be separated from the aorta wall in order to construct the $3 \mathrm{D}$ shape model. First of all, the inner boundary is separated from the outer one based on Fig. 5 (b). The separated boundaries are shown in Fig. 9. Then, the inner boundary (Fig. 9 (b)) is expanded until it reaches the outer one shown in Fig. 9 (a). Fig. 10 (a) is the expanded image and the abstract aorta wall image is obtained by intersecting the expanded image (Fig. 10 (a)) and the original binary image (Fig. 4 (b)). The intersection result is shown in Fig. 10 (b).

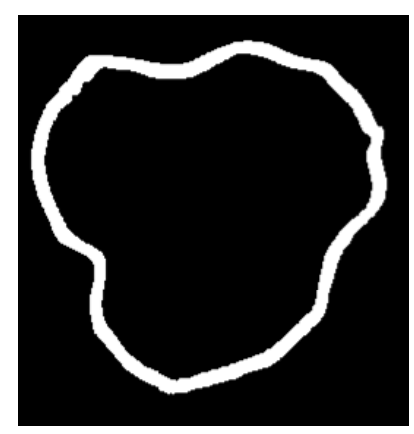

Figure 8. Estimated wall (horizontal cross section).

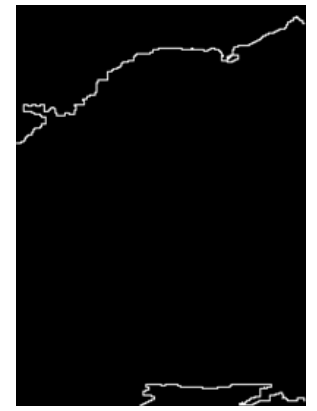

(a) outer boundary

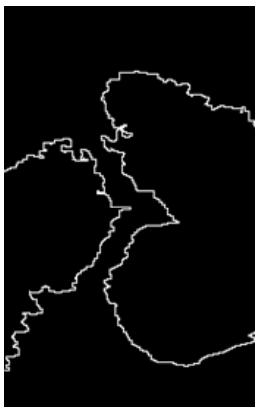

(b) inner boundar

Figure 9. Outer and inner boundaries.

This time, the same process is applied to the outer boundary. That is, the outer boundary (Fig. 9 (a)) is expanded with the same count as the number for the inner boundary. The expanded image is shown in Fig. 11 (a), and the aorta wall is separated from the aorta valve by intersecting the expanded image (Fig. 11 (a)) and the previous intersection image (Fig. 10 (b)). Then, the original image (Fig. 1 (b)) is binarized with the threshold 200 , and the same process as one for the horizontal cross section is applied to the vertical cross section, where the half line is extended from the inner boundary points to the outer one. The measurement process and the estimated wall image are shown in Fig. 12 and Fig. 13, respectively.

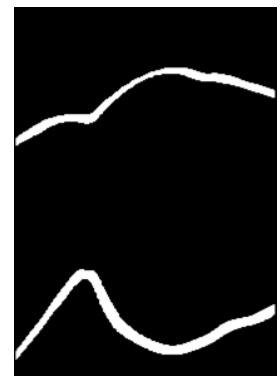

Figure 13. Estimated wall (vertical cross section).

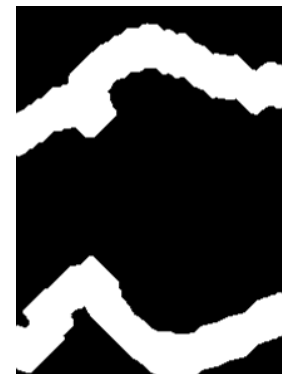

(a) outer expansion

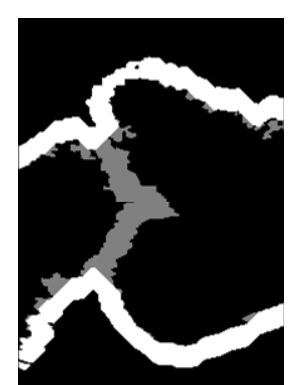

(b) separation

Figure 11. Inner expansion and separation.

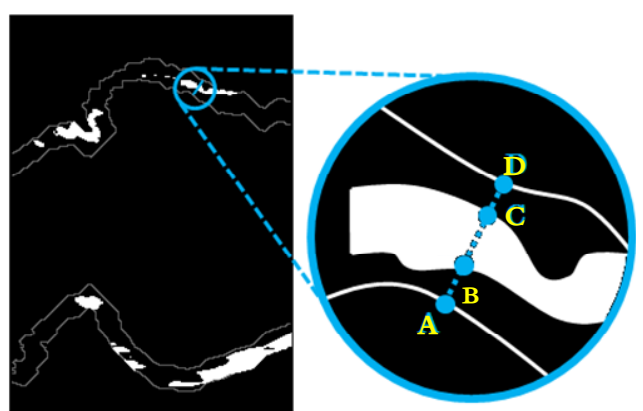

Figure 12. Thickness measurement (vertical). 


\section{ESTIMATION OF AORTA VALVE}

\subsection{Center of the aorta valve}

In section 3.1 , a temporary center was defined with an inscribed maximum triangle. In this section, the actual center of the aorta valve is defined with the temporary center. The length between the temporary center and each point constructing the inner boundary is measured and three points that give the minimum lengths among them are selected. Then, the inner boundary of the aorta can be divided into three parts with the three points. Then, each part is approximated with an ellipse by least squares method. Fig. 14 (a) shows the three approximated ellipses, and the actual center of the aorta is defined as the center of gravity for the intersection of the three ellipses. With the defined actual center, the aorta valve is divided into three elements (See Fig. 14 (b)).

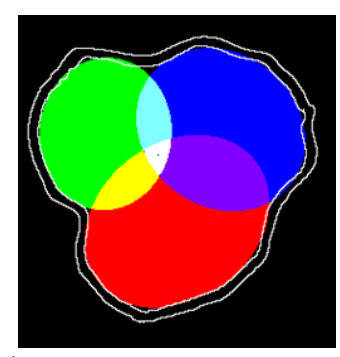

(a) three approximated ellipses

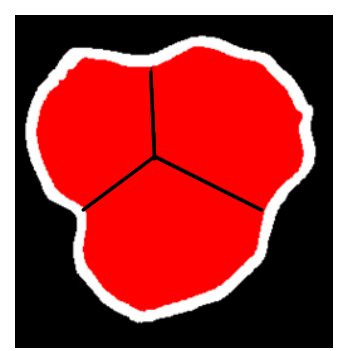

(b) three divided elements of the aorta valve

Figure 14. Ellipse approximation and the aorta division.

\subsection{Shape of the aorta valve}

In section 3.2, the aorta valve was separated from the wall so that the shape of the aorta valve can be estimated with the same method. The separated aorta valve and the contour are shown in Fig. 15 (a) and (b), respectively. However, the wall is too thick as mentioned in section 3.1. Then, the binarization with the threshold 200 is applied to the original image (Fig. 1 (a)), and the obtained binary image is intersected with the image of Fig. 15 (a). The result is shown in the left image of Fig. 16 and the thickness of the wall is estimated with the same method as described in section 3.2. The measurement process and the estimated valve image are shown in the right image of Fig. 16 and Fig. 17, respectively.

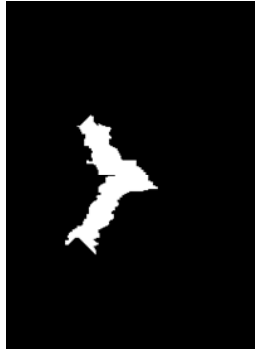

(a) aorta valve

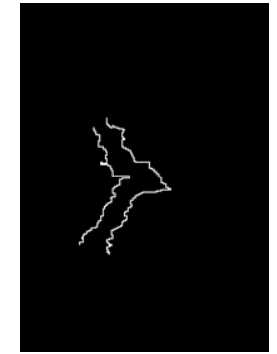

(b) contour of the valve

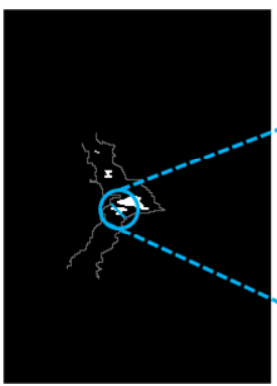

Figure 16. Thickness measurement (valve).

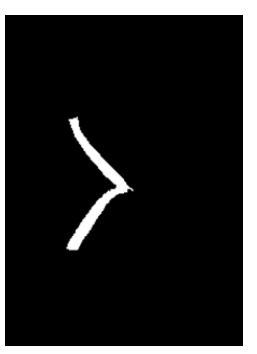
valve.
Figure 15. Separated aorta valve and its contour.
Figure 17. Estimated

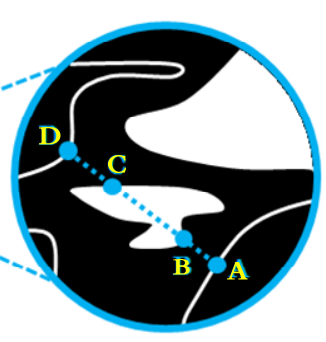

\section{CONSTRUCTION OF 3D AORTA SHAPE MODEL}

\subsection{Insertion direction}

With the above methods, we have obtained three images: the horizontal and the vertical cross sections of the aorta wall, and the aorta valve. Basically, we can construct the 3D aorta model by inserting the horizontal wall and the valve images into the vertical wall image. Here, there are two points to keep in mind. One is the direction in which the horizontal wall or the valve images are inserted, and the other is the image size. The obtained image is just one sample of the horizontal wall or the valve so that the inserted image should be generated by expanding or shrinking the obtained image.

The Fig. 18 (a) and (b) show the insertion directions for the aorta wall and the valve images, respectively. In Fig. 18 (a), (1), (2), (3) and (4) are the four end points of the aorta wall in the image, and (5) and (6) are the middle points of them (5) is the middle point of (1) and (2), and (6) is the middle point of (3) and (4)). The insertion direction for the aorta wall is perpendicular to the line that connects (5) and (6). On the other hand, in Fig. 18 (b), (1), (2), (3) and (4) are the four points that connect the aorta wall and the valve. Then, the 
insertion direction for the aorta valve is parallel to the line that has the average slope of the two lines; one is the line connecting (1) and (2), and the other is the line connecting (3) and (4).

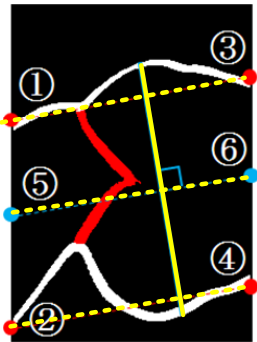

(a) direction of the wall

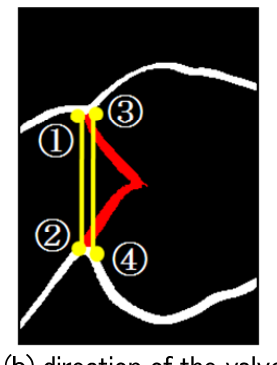

(b) direction of the valve

Figure 18. Insertion directions.

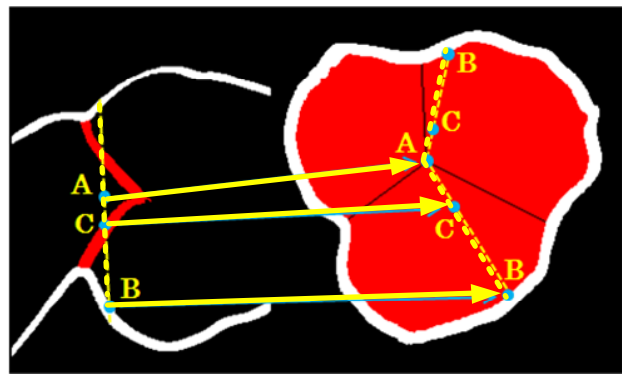

Figure 19. Image customization.

\subsection{Image customization}

For the insertion of both the aorta wall and the valve images, the image customization is necessary, which means the image should be expanded or shrunk according to the vertical aorta wall image. Fig. 19 illustrates how to calculate the image size. In Fig. 19, A is the center point of the aorta valve in the image, and $\mathrm{B}$ is an inner point of the aorta wall. The right image of Fig. 19 shows two inner points of the aorta wall; however, there are many inner points. In addition, $\mathrm{C}$ is the point on the aorta valve. Then, the image is customized, where $\mathrm{AC} / \mathrm{AB}$ in the right image equals to the $\mathrm{AC} / \mathrm{AB}$ in the left one.

Fig. 20 shows three examples of the insertion positions and the customized images. Fig. 20 (b) and (d) are the most outer and the most inner parts of the aorta valve respectively, and Fig. 20 (c) is the middle part. The aorta valve is divided into three elements so that each customized image has three gaps that correspond to the three elements of the valve.

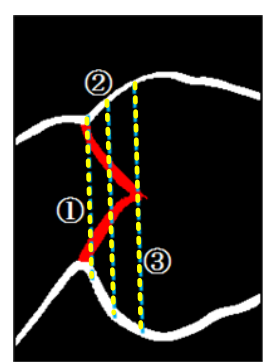

(a) insert position

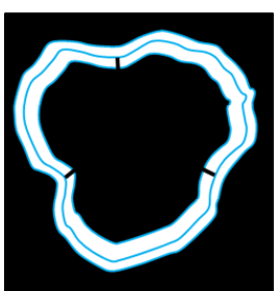

(b) image for (1)

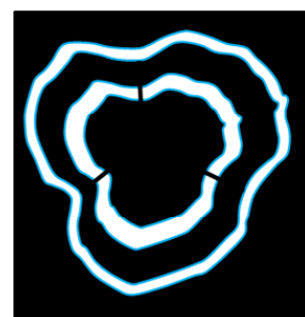

(c) image for (2)

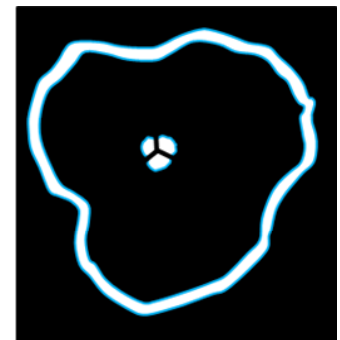

(d) image for (3)

Figure 20. Examples of the customized images.

\subsection{Result}

By inserting the customized horizontal images, which are obtained above, into the vertical cross section image of the aorta wall, the 3D shape model of the aorta can be constructed. Fig. 21 shows the abstract image of the insertion and Fig. 1 (c) and (d) show the constructed 3D shape model of the aorta. In addition, Fig. 22 shows the comparison of the constructed image with the original image. The original image (Fig. 22 (a)) has

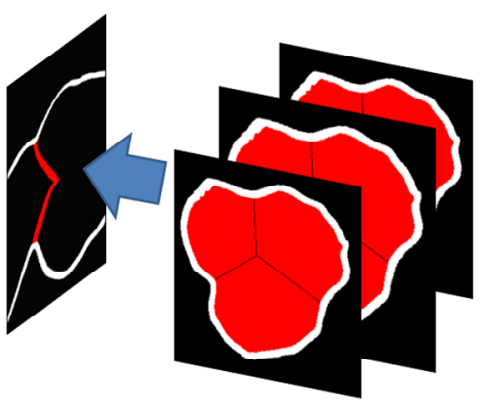

Figure 21. Insertion of images.

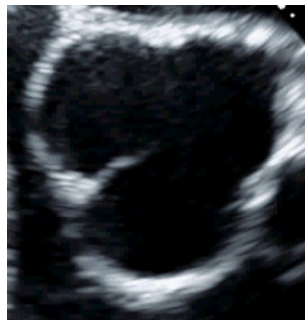

(a) original image

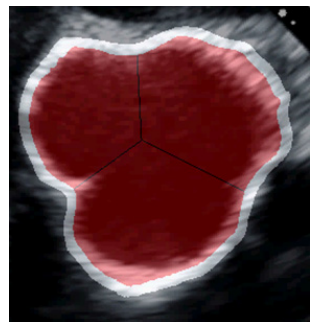

(b) overlap image
Figure 22. Comparison of the original and the constructed images. 
many noises and some echo that reflects some parts around the aorta wall; however, the 2D image of the constructed 3D aorta model has the essential thickness of the aorta wall and the clear valve image.

\section{DISCUSSION AND CONCLUSIONS}

In this paper, we have suggested the construction method of 3D aorta model from 2D US images, which are the images of the horizontal and the vertical cross sections. In order to construct the model, the original images were binarized to obtain the boundary, and the thickness of the aorta wall was estimated based on another binarized image after a temporary center had been decided. The estimation was necessary for the horizontal and the vertical cross section images and also the aorta valve. Then, the actual center was calculated with least squares method for three ellipses. Finally, the 3D aorta shape model was constructed by defining the insertion directions and by customizing the inserted images for the vertical cross section image. The constructed 3D model has three clear aorta valve elements and no noise when it is compared with the original image.

The constructed 3D shape model has some volume so that we can see it from any point of view by rotation, and we also can use it for some medical applications. The construction method is performed almost automatically; however, there are some parameters defined by manual, such as threshold of binarization, measurement of thickness, insertion direction of the horizontal cross section images to the vertical one, and so on. These are some issues to be solved.

In the near future, we have to improve our system by reducing the parameters defined by manual. We also plan to use the constructed 3D aorta model for surgery simulations or preoperative plannings. Especially, in the surgery of AAE, it is necessary to search how strong stress are there in the aorta valve. For this purpose, we have to simulate the blood stream in the aorta and calculate the stress on the aorta valve. In order to solve this issue, we think that particle method is one approach. By expressing the all materials including not only the blood itself but also the blood vessel as particle model, it is possible to calculate the interaction between the blood vessel and the blood with a uniform method. Therefore, we plan to simulate the blood stream in the aorta with the constructed 3D aorta model and to visualize the stress on the aorta valve.

\section{ACKNOWLEDGMENTS}

This research was supported by Japan Society for the Promotion of Science (Research No.21500125).

\section{REFERENCES}

Aoyama, M., T. Kusu, M. Mukunoki, N. Asada, Y. Komeda, T. Okigawa, and J. Urata (2010). Intracranial Aneurysms Detection Using Directional Vector of Luminance Distribution in MRA Images. The Transactions of the Institute of Electronics, Information and Communication Engineers D, J93(2), 139147.

Chrisochoides, N., A. Fedorovt, and A. Kot (2006). Toward Real-Time Guided Neurosurgery Using Distributed and Grid Computing. Proceedings of the $6^{\text {th }}$ ACM SIGMM International workshop on Multimedia information retrieval, 135-142.

Glatard, T., J. Montagnat, and I. E. Magnin (2004). Texture Based Medical Image Indexing and Retrieval: Application to Cardiac Imaging. Proceedings of the 2006 ACM/IEEE Conference on Supercomputing, 13 pages.

Oda, N., S. Kido, H. Shouno, and K. Ueda (2004). Development of Computerized System for Detection of Pulmonary Nodules on Digital Chest Radiographs Using Temporal Subtraction Images. The Transactions of the Institute of Electronics, Information and Communication Engineers D-II, J87(1), 208-218.

Plesniak, W., M. Halle, S. D. Pieper, W. Wells III, M., Jakab, and D. S. Meier (2003). Holographic Video Display of Time-Series Volumetric Medical Data. Proceedings of the $14^{\text {th }}$ IEEE Visualization, 589-596.

Tatefuku, Y., N. Mukai, K. Niki, and S. Takanashi (2010). Generation Method of 3D Aortic Shape Model Based on 2D Ultrasound Echo images. Proceedings of the Society of Instrument and Control Engineers (SI2010), 2129-2132.

Toosi, T. K. and H. Behnam (2009). Combined Pulse Compression and Adaptive Beamforming in Coded Excitation Ultrasound Medical Imaging. Proceedings of 2009 International Conference on Signal Processing Systems, 210-214.

Trucco, A., S. Curletto, and M. Palmese (2009). Interpolation of Medical Ultrasound Images from Coherent and Noncoherent Signals. IEEE Trans. on Instrumentation and Mesurement, 58(7), 2048-2060. 